\title{
Haus- und Kinderärzte belegen Spitzenplatz in der Zusammenarbeit
}

\author{
Nathalie Bösch ${ }^{a}$, Esther Kraft ${ }^{a}$, Clémence Merçay ${ }^{b}$, Paul Camenzind ${ }^{b}$ \\ a $\mathrm{FMH} ;{ }^{b}$ Obsan
}

* Zur besseren Lesbarkeit wird-wenn nicht ausdrücklich erwähnt - die männliche Schreibweise verwendet.

\footnotetext{
1 USA, Deutschland, Niederlande, Kanada, Grossbritannien, Australien, Norwegen, Neuseeland, Schweden. Die Daten zum ebenfalls teilnehmenden Frankreich lagen leider zur Zeit der Redaktion dieses Textes noch nicht vor.
}

\author{
Seit 2010 nimmt die Schweiz am internationalen Health Policy Survey der ame- \\ rikanischen Stiftung "Commonwealth Fund» teil. Zum zweiten Mal wurden die \\ ärztlichen Grundversorger/-innen in der Befragung 2015 in den Fokus gestellt und \\ international verglichen.
}

Die ärztliche Grundversorgung ist in vielen Ländern von hoher Aktualität. Der Commonwealth Fund [1] stellt deshalb in dreijährigem Rhythmus die Grundversorgerinnen und Grundversorger ins Zentrum seiner Befragung. Beim Commonwealth Fund handelt es sich um eine gemeinnützige amerikanische Stiftung, die seit 1998 jährlich international vergleichende gesundheitspolitische Befragungen durchführt. Das Bundesamt für Gesundheit BAG und die FMH führten 2015 zum zweiten Mal gemeinsam die Befragung der Grundversorger* in der Schweiz durch, das Obsan übernimmt jeweils die Auswertung und Analyse der Ergebnisse. Die meisten Fragen wurden den Grundversorgerinnen und Grundversorgern aller teilnehmenden Länder ${ }^{1}$ gestellt. In Zusammenarbeit mit «Hausärzte Schweiz» wurden für die Schweiz einige Fragen ergänzt, die sich speziell auf die hiesige Situation beziehen.

Bei der Umfrage 2015 durch ein unabhängiges externes Institut nahmen 1064 von 2734 angeschriebenen Grundversorgern teil (69,6\% aus der Deutschschweiz, 25,6\% aus der Romandie und 4,8\% aus dem Tessin). Zwei Drittel $(68,5 \%)$ der Befragten sind zwischen 45 und 64 Jahre alt, der Anteil der Frauen liegt bei 33\%, jener der Männer bei $67 \%$. Die Stichprobe entspricht grundsätzlich der Grundgesamtheit der ärztlichen Grundversorgerinnen und Grundversorger in der Schweiz. Nur die Ärzte in Gruppenpraxen waren übervertreten. In der Schweiz haben vergleichsweise viele Ärzte an der Umfrage teilgenommen. So waren es in den Vereinigten Staaten lediglich 1001 teilnehmende Ärzte, was im Vergleich zur dortigen Anzahl Grundversorger nicht sonderlich hoch ist.

Die Fragen des Commonwealth Fund International Health Policy Survey 2015, erweitert durch die erwähnten landesspezifischen Ergänzungen, betrafen eine Vielzahl von Themen wie z.B. Zufriedenheit mit dem Gesundheitssystem, Zugang zu den Gesundheitsleistungen oder Zusammenarbeit mit anderen ärztlichen und medizinischen Leistungserbringern. Die nachfolgenden Ausführungen sind ein Auszug aus den umfassend publizierten Resultaten [2]. Bei der Interpretation der internationalen Daten müssen die länderspezifischen Eigenschaften des jeweiligen Gesundheitssystems mit berücksichtigt werden.

\section{Schweizer Resultate im internationalen Vergleich}

Die Resultate der zweiten internationalen Befragung des Commonwealth Funds von ärztlichen Grundversorgern in zehn Ländern zeigen, dass Hausärzte und Pädiater mit einem hohen administrativen Aufwand belastet sind. Ausserdem verdeutlichen sie den ärztlichen Nachwuchsmangel in unserem Land. In der Koordination der Behandlungsbeteiligten schneidet die Schweiz im internationalen Vergleich am besten $\mathrm{ab}-$ mit eindeutig positiven Auswirkungen auf die Patienten aus ärztlicher Sicht.

\section{Allgemeine Steigerung der Zufriedenheit der Ärzte in ihrer Tätigkeit trotz hoher Arbeitsbelastung}

Die Zufriedenheit der befragten Grundversorger mit ihrer beruflichen Tätigkeit stieg im Vergleich zum Jahr 2012 leicht an (Abb. 1) und es herrscht eine positive Einstellung gegenüber dem Schweizer Gesundheitssystem. Auch im internationalen Vergleich sind die Schweizer Grundversorger zufrieden in der Ausübung ihres Berufes. Im Jahr 2015 waren weniger Grundversorger der Meinung, dass grössere Anpassungen im Gesundheitssystem notwendig wären, als noch vor drei Jahren. Der Prozentsatz sank von 49\% auf 44\%. Trotz der Abnahme der wöchentlichen Arbeitsstunden liegt die Schweiz hinter Deutschland bei der Arbeits- 


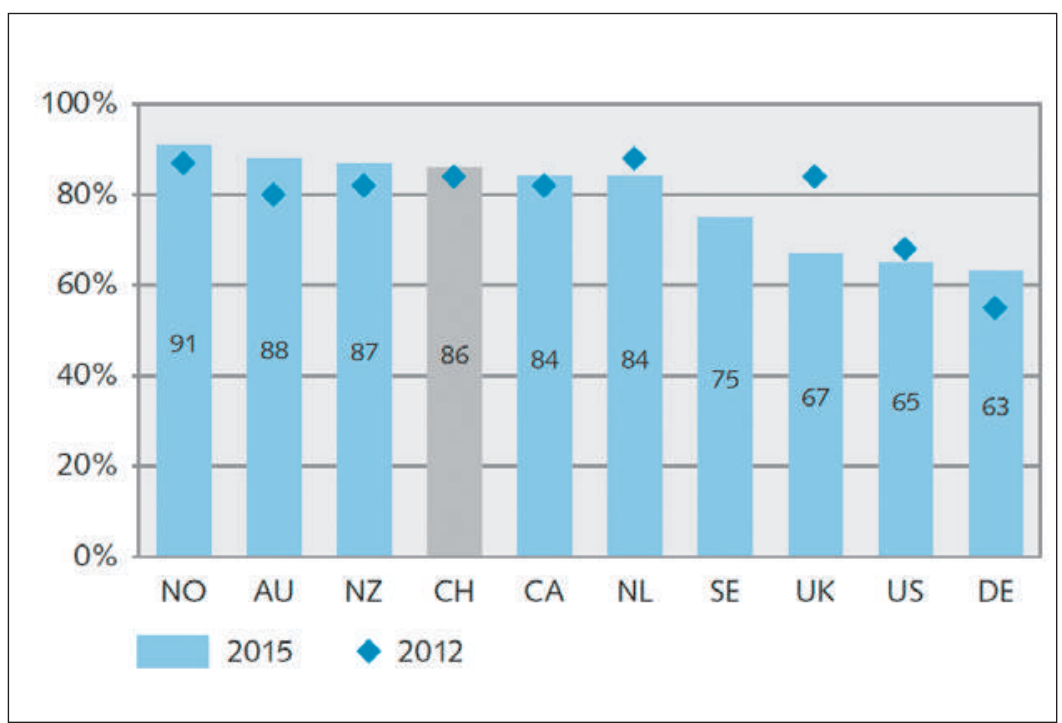

Abbildung 1: Zufriedenheit der Ärzte mit der Berufsausübung im internationalen Vergleich. Quelle: The Commonwealth Fund, International Health Policy Survey 2015.

belastung auf Platz 2 im internationalen Vergleich. $60 \%$ der Grundversorger arbeiten mehr als 45 Stunden pro Woche. 2012 hatte dieser Prozentsatz noch bei 68\% gelegen.

\section{Positive Ergebnisse bei der Kommuni- kation mit Patienten, den anderen Spezialärzten und den Spitälern}

In der Zusammenarbeit zwischen ärztlichen Grundversorgern und den Diensten der Pflege und Hilfe zu Hause (in der Schweiz die "Spitex») belegt unser Land im internationalen Vergleich den ersten Rang: Während 74 Prozent der Grundversorgerinnen in der Schweiz sehr zufrieden sind und die Koordination mit der Spitex als «leicht» oder "sehr leicht» beschreiben,

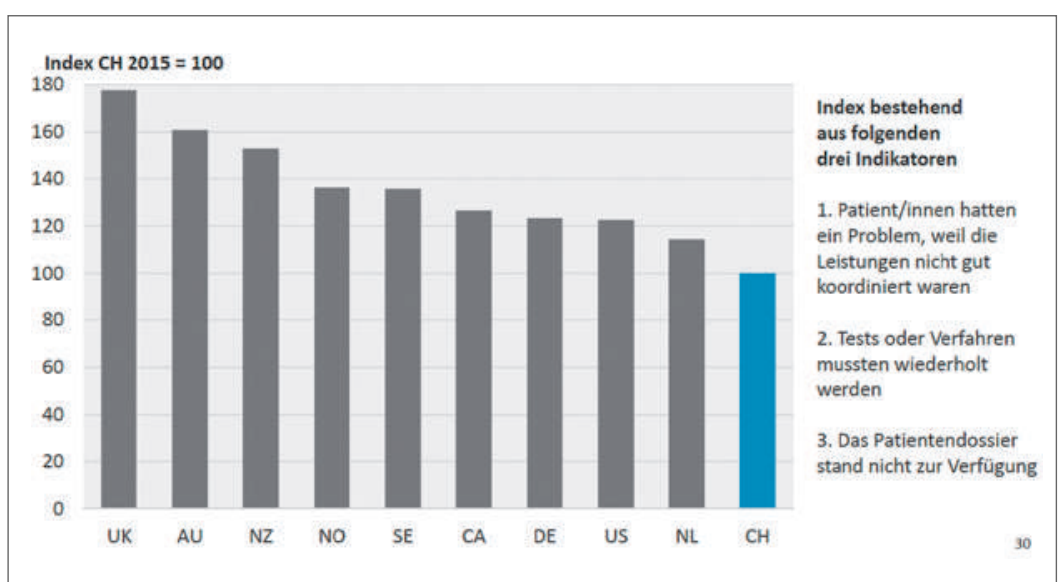

Abbildung 2: Index zur Problementstehung durch schlechte Koordination im internationalen Vergleich. Quelle: The Commonwealth Fund, International Health Policy Survey 2015 . liegt der Durchschnitt der anderen Länder bei 38 Prozent.

Die Koordination zwischen Grundversorgern und den Spezialärzten funktioniert ebenfalls sehr gut: Beinahe alle Grundversorger (94 Prozent) geben an, dass sie «immer» oder «oft» einen Bericht vom Spezialisten erhalten, der alle relevanten Gesundheitsinformationen enthält. Auch bei der Zusammenarbeit mit den Spitälern bewegt sich die Schweiz im oberen Mittelfeld: 82 Prozent der Grundversorger erhalten «immer» oder «oft» Informationen über Krankenhausaufenthalte ihrer Patienten. Diese positiven Ergebnisse zeigen sich auch darin, dass es für Patienten selten zu Problemen wegen schlechter Koordination kommt (Abb. 2).

Die Schweizer Patienten können sich im internationalen Vergleich über sehr kurze Wartezeiten erfreuen. Die Schweiz nimmt auch hier 2015 die führende Position ein. Auch die Verfügbarkeit der Grundversorger ist gut, obwohl seit 2012 sinkende Trends zu verzeichnen sind. 2015 können 78\% (2012: 86\%) der ärztlichen Grundversorger noch neue Patienten aufnehmen und 54\% (2012: 62\%) der Patienten mit Bedarf an einer kurzfristigen Konsultation kann diese auch angeboten werden. Für die Patienten bedeutet dies, dass ihnen im internationalen Vergleich am seltensten Probleme wegen mangelnder Koordination der Behandlungsbeteiligten widerfahren. Auch unnötige Testwiederholungen wegen nicht-vorliegender Resultate aus früheren Untersuchungen treten in der Schweiz am seltensten auf.

Die ärztlichen Grundversorger zeigen sich im Allgemeinen eher skeptisch gegenüber neuen, aktuell diskutierten Instrumenten (z.B. Disease Management, Case Management) zur Verbesserung der Zusammenarbeit. 2015 haben 65\% diesbezüglich drei oder mehr Vorbehalte geäussert. Diese Skepsis gegenüber den neuen Instrumenten dürfte bestimmt auch an der grossen Zufriedenheit der Grundversorger mit der jetzigen Situation und der guten Position im internationalen Vergleich liegen. Die Grundversorger sehen bei den hohen Kosten die grösste Problematik.

\section{Weniger Zeit für den Patienten wegen administrativer Aufgaben}

Jeder zweite ärztliche Grundversorger nimmt den mit Versicherungen verbundenen administrativen Aufwand als grosses Problem wahr, jeder Dritte betrachtet auch den Aufwand für die Erfassung klinischer Daten oder das Sammeln von Qualitätsdaten für staatliche oder andere Institutionen als problematisch. Im Vergleich zur Befragung 2012 nahm der Anteil der Grundversorger, die mindestens 75 Prozent ihrer Arbeitszeit 
mit Patientenkontakten verbringen können, deutlich ab: von 60 Prozent der Grundversorger im Jahr 2012 auf nur noch 45 Prozent im Jahr 2015. Diese Tendenz entspricht auch den Ergebnissen anderer Studien, die eine Zunahme der administrativen Belastung zeigen.

\section{Raum zur Verbesserung bei der Nutzung von eHealth-Instrumenten}

Die Schweiz konnte zwar in den letzten drei Jahren eine grössere Verbreitung von elektronisch erfassten Krankengeschichten (von $41 \%$ auf 54\% der Ärzte) verzeichnen, jedoch bildet sie im internationalen Vergleich immer noch das Schlusslicht. Es ist auch eine sehr ungleiche Verbreitung festzustellen. Vor allem bei den Grundversorgern, die jünger als 45 Jahre alt sind, und in den Gruppenpraxen werden elektronisch erfasste Krankengeschichten häufiger genutzt. Auch gibt es regionale Unterschiede. In der Deutschschweiz sind sie am weitesten verbreitet, im Tessin am wenigsten.

Es sind jedoch auch nicht alle eHealth-Instrumente gleich verteilt. So können bereits $80 \%$ der Grundversorger ihren Patienten die Möglichkeit, per E-Mail Kontakt aufzunehmen, anbieten. Auch bei der elektronischen Kommunikation mit Labors und anderen Spezialärzten zeigten sich Verbesserungen. Hingegen sind nur $24 \%$ in der Lage, elektronische Medikamentenverschreibungen bei Apotheken durchzuführen. Bei der Übermittlung von Daten und Informationen zwischen Grundversorgern und Spitälern bleibt das Faxgerät mit 41\% das meistgenutzte Instrument. Nur 19\% nutzen hier E-Mails für den Austausch von Informationen.

\section{Allgemeiner Nachwuchsmangel}

In der Schweiz herrscht grosser Nachwuchsmangel bei den ärztlichen Grundversorgern. Rund ein Drittel der Schweizer Grundversorger ist 60 Jahre alt oder älter und lediglich $20 \%$ von ihnen haben einen Nachfolger für ihre Praxis. Der heutige Nachwuchsmangel lässt sich von zwei Seiten betrachten. Erstens lässt er sich erklären durch die geringe Anzahl an Medizinstudienplätzen in den letzten 20 Jahren. Die Politik hat inzwischen erkannt, dass Änderungen notwendig sind, und die Zahl der Studienplätze wurde erhöht. Bis die heutigen Studienanfänger selbständig praktizieren können, werden jedoch rund 12 Jahre vergehen. Somit hat die Schweiz vor allem in den nächsten Jahren grosse Herausforderungen bei der Abdeckung des Bedarfs an Grundversorgern zu bewältigen.

Die zweite Sichtweise betrifft die Attraktivität des Be- rufes als ärztlicher Grundversorger. Sowohl das Lohnniveau der Grundversorger im Vergleich zu den Löhnen anderer Spezialisten als auch die hohe administrative Belastung dürften ebenfalls relevante Faktoren sein, die das Interesse an der Ausübung ärztlicher Grundversorgung beeinflussen. Dabei sind die Schweizer Grundversorger heute zufriedener mit ihrem Einkommen als vor drei Jahren. 2015 gaben $72 \%$ an, zufrieden zu sein, während dies 2012 nur 57\% gewesen waren. Jedoch zeigt sich 2015, wie auch schon 2012, eine grosse Unzufriedenheit beim Einkommensvergleich zu Spezialärzten: nur 19\% der Befragten zeigten sich hier zufrieden.

Nachwuchsmangel in der Grundversorgung als grosse Herausforderung der kommenden Jahre.

\section{Schlussbemerkung}

Die Schweizer Grundversorger sind mit ihrer ärztlichen Tätigkeit zufrieden und nehmen im internationalen Vergleich generell eine gute Position ein. Die Zufriedenheit konnte auch im Vergleich zum Jahr 2012 noch gesteigert werden. Vor allem bei der Kommunikation und Koordination zwischen Grundversorgern und Spezialisten sowie mit Spitex-Organisationen sind die Schweizer Ärzte führend. Aber auch bezüglich kurzer Wartezeiten und Verfügbarkeit der Grundversorger ist das Ergebnis sehr gut.

Ein Element, mit dem die Grundversorger in der Schweiz gar nicht zufrieden sind, ist die Höhe des eigenen Lohns im Vergleich zum Lohn der Spezialisten. Zwei weitere Probleme werden bei einer übermässigen Menge an Leistungen für die Patienten sowie beim zu grossen administrativen Aufwand gesehen. Weiter gibt es in der Schweiz, international gesehen, immer noch sehr lange Arbeitszeiten; und dies trotz rückläufiger Tendenzen. Verbesserungspotential besteht bei Management-Instrumenten der Praxistätigkeit (Praxisindikatoren und -vergleiche) sowie bei der Nutzung und Verbreitung von eHealth-Instrumenten. Die grössten Herausforderungen der nächsten Jahre bleiben aber die Ärztedemographie der Schweiz und der Nachwuchsmangel.

Diese Ausführungen sind ein Auszug aus den umfassend publizierten Ergebnissen des Commonwealth Fund International Health Survey von 2015 [2]. Die FMH steht Ihnen gerne für weitere Auskünfte zur Verfügung unter ddq[at]fmh.ch oder 0313591111.

\footnotetext{
Literatur

1 www.commonwealthfund or

2 www.bag.admin.ch/themen/gesundheitspolitik
} 